\title{
Bi-articular muscles and the accuracy of motor control
}

\author{
Jeroen B.J. Smeets * \\ Dept. of Physiology, Erasmus University, P.O. Box 1738, 3000 DR Rotterdam, The Netherlands
}

\begin{abstract}
In a model study, the behaviour of two sets of muscles in controlling multi-joint arm movements is compared. Both the sensory and the motor accuracy of the set containing bi-articulat muscles were in general better than those of the set containing only mono-articular muscles. Accuracy considerations can explain differences in strategies for the control of redundant muscle sets between situations which do not differ biomechanically from each other. Furthermore, the role of bi-articular muscles for the robustness of motor programming is discussed.
\end{abstract}

\section{Introduction}

Theories on muscle coordination in general, and thus also theories on the special role of bi-articular muscles, focus on the force, displacement and related variables (as work, energy) caused by activating muscles. They tend to concentrate on one of these aspects and try to find an optimum solution for the control of that variable. However, the variable controlled (and thus the control strategy) could depend on the nature of the task (Stein, 1982; Tax et al., 1990). Sensory information from muscle afferents seems to play a crucial role in the ability to adapt movements to different environments, as can be concluded from studies on deafferented patients (Rothwell et al., 1982; Ghez et al., 1990). It seems a good hypothesis to

\footnotetext{
*Corresponding author: E-mail: smeets@fgg.eur.nl; Fax: + 3110 4367-594; Tel.: +31 10 4087-565.
} 


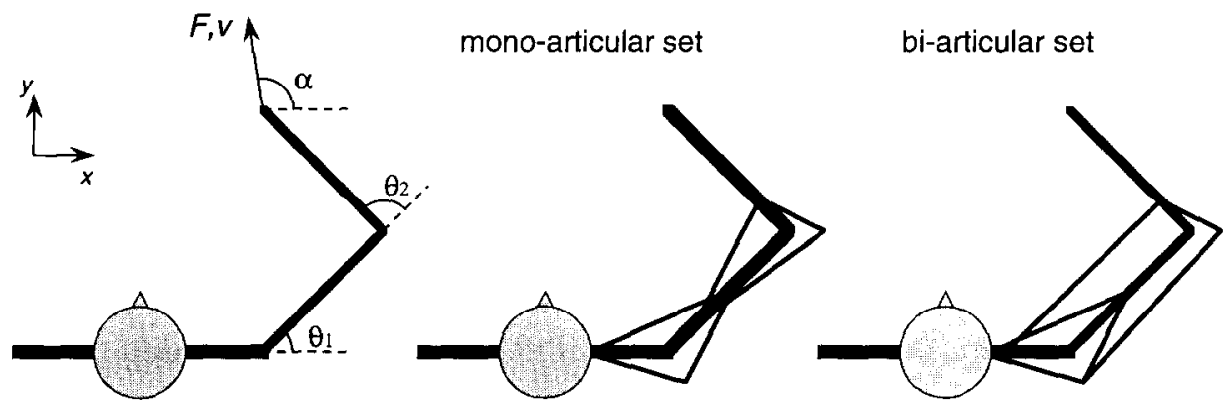

Fig. 1. The configuration of the (planar) arm as used in this paper, with the definitions of angles and directions. For the two sets used the muscles are indicated.

assume that also the accuracy of sensory information plays an important role in normal motor control. In this paper, the dependence of the accuracy of sensory and motor information on the bi-articularity of muscles will be studied. It will be shown that this dependence will cause additional constraints on muscle coordination: not only the variable controlled, but also accuracy requirements for that variable will affect the optimality of muscle coordination.

Movements of an arm in a plane (Fig. 1) will be used as an example. The role of bi-articular muscles is investigated by comparing the behaviour of two sets of muscles to move the arm. The mono-articular set consists of antagonistic muscle pairs around elbow and shoulder; in the bi-articular set, the antagonistic pair around the elbow is replaced by a bi-articular antagonistic pair. In this way, both sets have the same amount of muscles; adding more muscles can always improve the performance.

\section{Sensory aspects}

To move the hand to another position, the distance and direction of the movement have to be specified in one way or another. To make accurate movements, it is therefore important that neural signals (activity of motoneurones and afferents) can be transformed accurately into movement of the hand (and vice versa). In this section, the velocity information conveyed by the Id-afferents is used as an example for the sensory information. This is of course not the only information necessary for making accurate 
movements, but it is an important one (Sittig et al., 1985). How do bi-articular muscles change the accuracy of this information?

To answer this question, we have to consider the geometry of the limb. If the arm moves with velocity $\vec{v}$ (speed $v$ in a direction $\alpha$, see Fig. 1 ), the joint velocities follow from the Jacobian transformation (e.g. Hogan, 1985):

$$
\dot{\vec{\theta}}=J^{-1} \dot{\vec{v}}
$$

For an arm configuration as in Fig. 1, with equal length $l$ for both upper and lower arm, this equation can be written as:

$$
\begin{aligned}
\left(\begin{array}{l}
\dot{\theta}_{1} \\
\dot{\theta}_{2}
\end{array}\right)= & \frac{v}{l \sin \theta_{2}}\left(\begin{array}{cc}
\cos \left(\theta_{1}+\theta_{2}\right) & \sin \left(\theta_{1}+\theta_{2}\right) \\
-\cos \theta_{1}-\cos \left(\theta_{1}+\theta_{2}\right) & -\sin \theta_{1}-\sin \left(\theta_{1}+\theta_{2}\right)
\end{array}\right) \\
& \times\left(\begin{array}{l}
\cos \alpha \\
\sin \alpha
\end{array}\right) .
\end{aligned}
$$

To find a sensory response as function of movement direction, the response characteristics of the muscle spindle afferents have to be modelled. For the present purpose, a very simple model suffices. The muscle spindle output is modelled as being proportional to velocity of muscle stretch, and zero for shortening muscles. Furthermore, the moment arms $r_{m j}$ of the muscles $m$ around joint $j$ are assumed to be equal (and constant) for all muscles: positive for flexors, negative for extensors and zero if a muscle does not cross a joint (see Table 1). The spindle output $s_{m}$ of muscle $m$ can be expressed as a function of joint velocities:

$$
s_{m}= \begin{cases}r_{m 1} \dot{\theta}_{1}+r_{m 2} \dot{\theta}_{2}, & \text { if } r_{m 1} \dot{\theta}_{1}+r_{m 2} \dot{\theta}_{2}>0, \\ 0, & \text { otherwise. }\end{cases}
$$

Combination of Eqs. (2) and (3) results in the output of muscle spindles as

\begin{tabular}{|c|c|c|c|c|c|}
\hline \multicolumn{3}{|c|}{ Mono-articular set } & \multicolumn{3}{|l|}{ Bi-articular set } \\
\hline Muscle & $r_{1}$ & $r_{2}$ & Muscle & $r_{1}$ & $r_{2}$ \\
\hline Pectoralis & 3 & 0 & Pectoralis & 3 & 0 \\
\hline Infraspinatus & -3 & 0 & Infraspinatus & -3 & 0 \\
\hline Brachialis & 0 & 3 & Biceps & 3 & 3 \\
\hline Triceps s.h. & 0 & -3 & Triceps 1.h. & -3 & -3 \\
\hline
\end{tabular}
a function of the speed $v$ and direction $\alpha$ of hand velocity.

Table 1

The two muscle sets used. Moment arms $\left(r_{1}\right.$, shoulder; $r_{2}$, elbow) are given in $\mathrm{cm}$ 

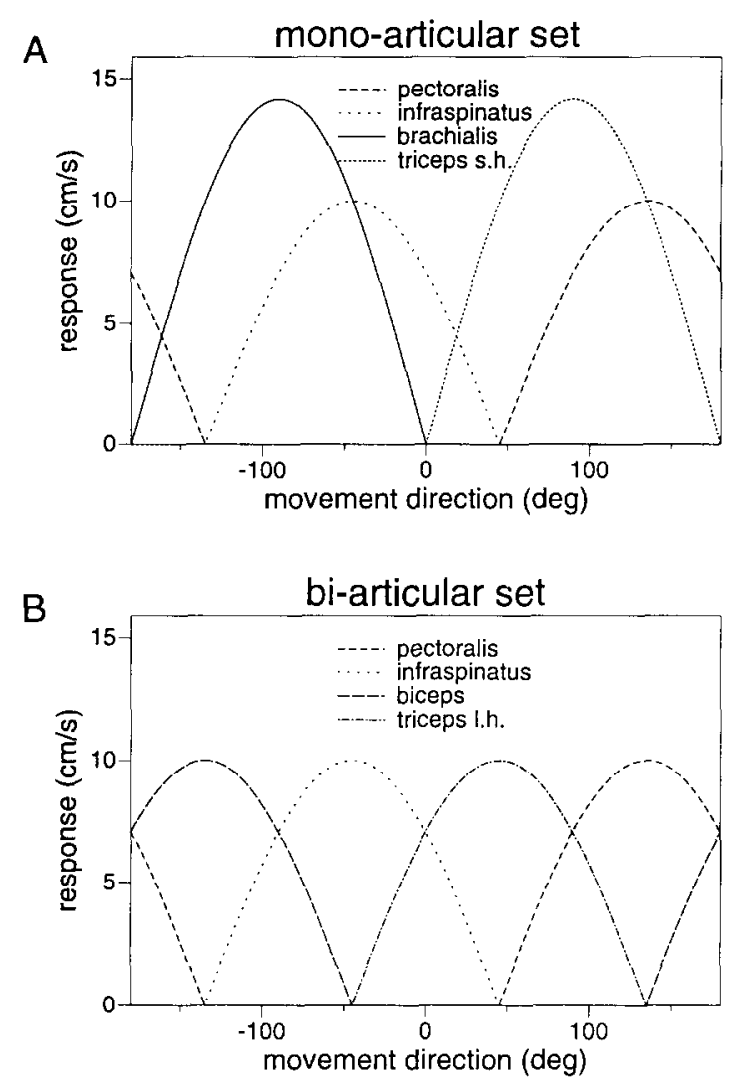

Fig. 2. The response $s_{m}$ of spindles in muscle $m$ as a function of the direction of movement. In each direction, a movement of the same speed is considered. A: The muscles of the mono-articular set. B: The muscles of the bi-articular set.

For the calculations, we chose a configuration as in Fig. 1: $\theta_{1}=45^{\circ}$ and $\theta_{2}=90^{\circ}$, and a velocity of $1 \mathrm{~m} / \mathrm{s}$. In Fig. $2 \mathrm{~A}$, the response of muscle spindles of the mono-articular muscle set is plotted for planar movements with equal velocities of the hand in various directions. The responses are broadly tuned: each spindle is sensitive for half of the possible movements. The maxima are not equally spread over the possible directions. The distances between the maxima depend on the elbow angle: for muscles around the shoulder the maximum response is for movements parallel to the forearm; for muscles around the elbow the maximum response is for movements in the direction of the hand-shoulder line. For all possible arm configurations, these positions of the maxima leave a gap in the responses 
between the directions of pure elbow-flexion and shoulder-flexion. Using the bi-articular set, this gap is in general smaller or absent (Fig. 2B). Why is the filling of the gap advantageous?

To understand the advantage of the bi-articular muscle, set, a more precise definition of an accurate transformation is needed. A reasonable definition seems to be "a transformation which is sensitive for differences in the measured variable in all movement directions, but insensitive for noise". The best overall behaviour is an output signal which changes smoothly with the variables of interest.

Using the output of the muscle spindles, the nervous system should be able to perceive both the speed and the direction of a movement accurately. How the nervous system solves or approximates the trigonometry of this task is unknown. The strategy is not to propose a scheme how the nervous system can measure speed and direction; instead, a very simple variable containing the required information is defined, and the effect of bi-articular muscles on the behaviour of this variable is studied.

The speed of a movement could be measured by the sum of the output $s_{m}$ of the muscle spindles. The speed sensitivity $S_{s}$ is thus

$$
S_{s}=\sum_{m} \frac{\partial s_{m}(\alpha, v)}{\partial v}
$$

It is clear from Eq. (2) and Eq. (3) that $S_{s}$ is independent of $v$ for any set of muscles; it should also be independent of the direction of movement. In Fig. 3A, the speed sensitivity is plotted as a function of movement direction for the mono-articular and bi-articular muscle sets. For the mono-articular muscle set, the sensitivity varies more with movement direction than for the bi-articular muscle set. This observation holds not only for the geometry of Fig. 1 and Table 1, but for a wide range of elbow-angles and moment arms $r_{i}$. For the set of moment arms in Table 1, all elbow angles smaller than $120^{\circ}$ yield similar results.

The direction of movement can be calculated from the differences between muscles in spindle output. The directional sensitivity is high as these differences change strongly with the direction of movement. The directional sensitivity can thus be defined as the sum of the absolute values of these changes in difference over all combinations $(m, n)$ of spindles:

$$
S_{d}=\sum_{m, n}\left|\frac{\partial s_{m}(\alpha, v)}{\partial \alpha}-\frac{\partial s_{n}(\alpha, v)}{\partial \alpha}\right| .
$$



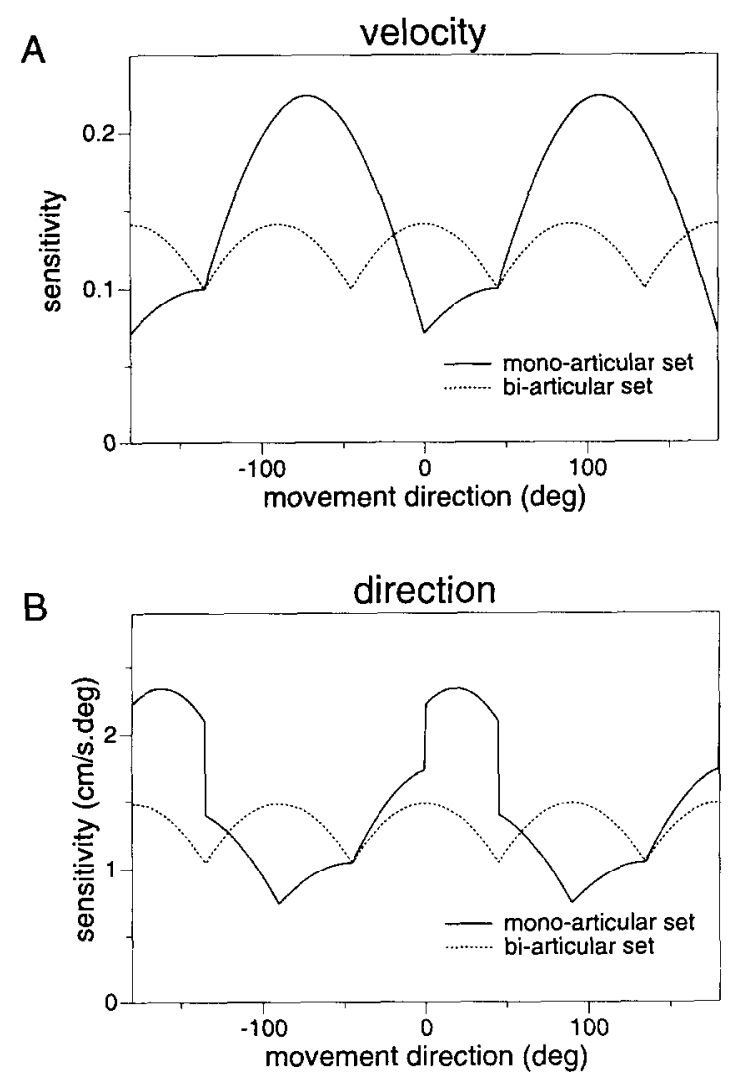

Fig. 3. The overall sensitivity of muscle spindles as a function of movement direction. Dotted lines: mono-articular muscle set, continuous lines: bi-articular muscle set. A: The (dimensionless) speed sensitivity $S_{s}$ (Eq. (4)). B: The directional sensitivity $S_{d}$ (Eq. (5)) for $1 \mathrm{~m} / \mathrm{s}$ movements.

It is clear from Eq. (2) and Eq. (3) that $S_{d}$ varies linearly with $v$ for any set of muscles. In Fig. 3B, the direction sensitivity is shown to be more constant for the bi-articular muscle set than for the mono-articular muscle set. Also this finding is robust for a wide range of moment arms and elbow angles (for the moment arms of Table 1: elbow angles flexed less than $\left.120^{\circ}\right)$.

So, one function of bi-articular muscles is to ensure a good overall accuracy of the sensory information about the speed and direction of a movement. 


\section{Motor aspects}

One could think that a similar reasoning as above could be given for the motor aspects of bi-articular muscles. This is however not true. Muscle spindle responses can be calculated straightforwardly if the movement is known, independent of the number of muscles which are crossing the joints. Calculating the muscle activities from a desired external force is a problem with not one single solution in general. If a limb has $N$ degrees of freedom, and more than $N+1$ muscles, the system is in general redundant: the same external force can be generated by an infinite amount of combinations of muscle activities. The arm of figure $1(N=2)$ for instance needs only three muscles to be able to generate external forces in all directions. Mono-articular flexors around elbow and shoulder combined with a bi-articular extensor are capable of generating a force in any direction. By using pairs of exact antagonists, we can increase the number of muscles to $2 \times N$ without introducing redundancy. As the two sets of muscles used in this paper (Fig. 1, Table 1) are non-redundant, we can compare the force-generating qualities of these sets.

To calculate the sensitivity, the transformations between muscle forces and forces in the external world have to be known. The activation $A_{m}$ of muscle $m$ will lead to moments $T_{j}$ around the joint $j$ :

$$
T_{j}=\sum_{m} A_{m} r_{m j}
$$

These moments lead to an external force $\vec{F}$ (with amplitude $F$ and direction $\alpha$ ), according to:

$$
\vec{F}=J^{-T} \vec{M}
$$

For the arm of Fig. 1 with equal length $l$ for both upper and lower arm, this equation can be written as:

$$
F\left(\begin{array}{c}
\cos \alpha \\
\sin \alpha
\end{array}\right)=\frac{v}{l \sin \theta_{2}}\left(\begin{array}{cc}
\cos \left(\theta_{1}+\theta_{2}\right) & -\cos \theta_{1}-\cos \left(\theta_{1}+\theta_{2}\right) \\
\sin \left(\theta_{1}+\theta_{2}\right) & -\sin \theta_{1}-\sin \left(\theta_{1}+\theta_{2}\right)
\end{array}\right)\left(\begin{array}{c}
T_{1} \\
T_{2}
\end{array}\right)
$$

For a non-redundant set of muscles, these equations can be used to construct the activation of muscles as a function of force direction. Activation of a mono-articular flexor of the elbow leads to a force in the direction of the shoulder; activation of a shoulder flexor leads to a force in the 

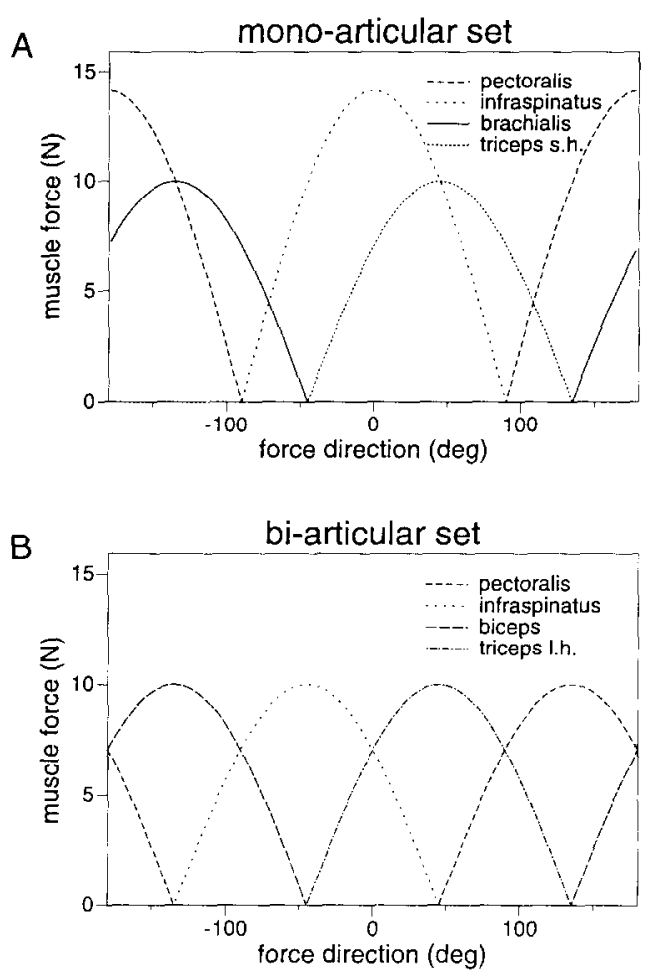

Fig. 4. The exerted force (activation $A_{m}$ ) of muscle $m$ to generate a $1 \mathrm{~N}$ external force as a function of force-direction. A: Mono-articular set. B: Bi-articular set.

direction of the lower arm. If we want to direct an equally strong force in between, we need activation of both mono-articular muscles, each at a higher level. This results in activity curves of the mono-articular muscle set as in Fig. 4A. In a redundant muscle set, bi-articular muscles generating moments of the same sign at both shoulder and elbow can take over a (large) part of the moments needed at both joints. In such a system, bi-articular muscles will cause a shift of the curves of the mono-articular muscles to the directions where they can generate the largest force. The amount of shift depends on the control strategy adopted (see next section).

For the non-redundant bi-articular muscle set, the resulting activation of the muscles as a function of force direction are plotted in Fig. 4B. It is directly clear that the activations of this set of muscles are better spread over the movement directions than those of the mono-articular set. Does 
this lead to a better performance? To answer this question, the accuracy of force generation has to be defined.

The accuracy of force generation can be defined in a similar way as in Eqs. (4)-(5). In force generating, variations in muscle force can be caused by for instance recruitment/decruitment, or fatigue. Variations are therefore considered which are proportional to muscle activation. A good accuracy means that small variations in muscle activity never lead to large variations in the amplitude or direction of force. The variation of direction and amplitude of force with small variations of muscle activity $A_{m}$ should be small and more or less constant, independent of force direction. The sensitivity of the amplitude of force for relative changes in muscle activation is:

$$
M_{a}=\sum_{m} A_{m}\left|\frac{\partial F}{\partial A_{m}}\right| .
$$

The sensitivity of the direction of force for variations in muscle activation is:

$$
M_{d}=\sum_{m} A_{m}\left|\frac{\partial \alpha}{\partial A_{m}}\right| .
$$

In Fig. 5A and $\mathrm{B}$, the resulting variabilities are plotted as functions of force direction. For the bi-articular muscle set, a change of muscle activation leads in each direction to the same change in force amplitude ( $1 \%$ change in activation leads to $1 \%$ force change), and in general a small change in force direction ( $1 \%$ change in activation leads to less than $0.5^{\circ}$ change in force direction). For the mono-articular muscle set, the changes in direction and amplitude of force due to variations in muscle activation are larger. So, the overall sensitivity for variations of the bi-articular muscle set is lower (higher accuracy) than that of the mono-articular set.

\section{Control strategies}

The effect of control strategies on motor-accuracy is neglected in the previous sections. The nervous system, however, can use the mono-articular muscle set or the bi-articular muscle set, or any linear combination of these to generate forces. An important result from the previous sections is that, for movements in some directions, the velocity perception and force 

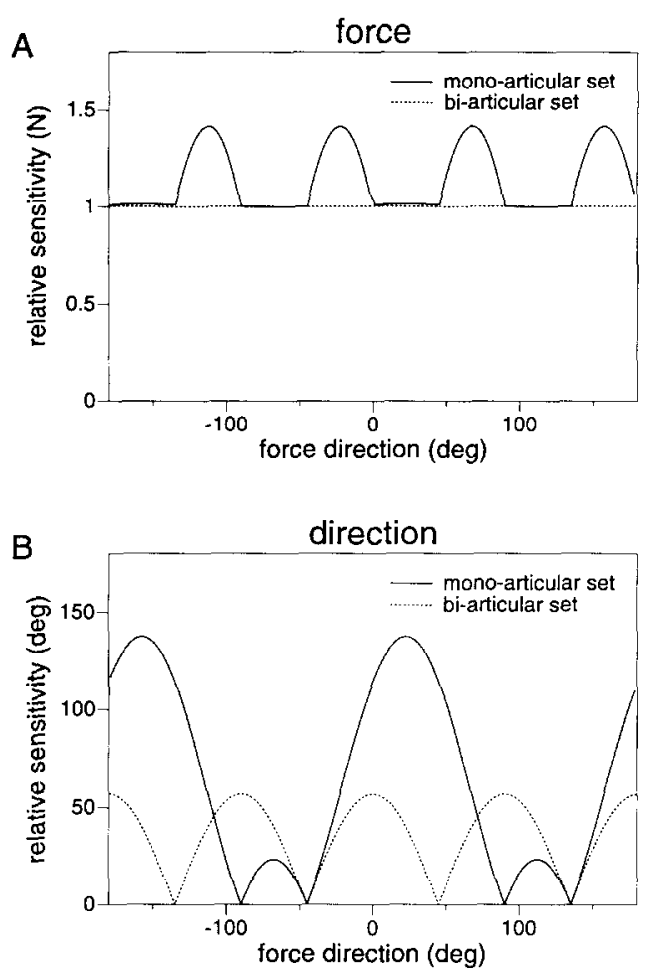

Fig. 5. The overall sensitivity of the external force for relative variations in muscle activation. A: The amplitude sensitivity $M_{a}$; a sensitivity of 1 corresponds to a $1 \%$ change in external force for a $1 \%$ change in muscle forces. B: The directional sensitivity $M_{d}$; a sensitivity of $100^{\circ}$ corresponds to a $1^{\circ}$ direction change for a $1 \%$ change in muscle forces.

generation are affected differently by the muscle set used (see Figs. 3 and 5). Control strategies using this phenomenon could optimize either the accuracy of the force, or the accuracy of a movement (or any weighted combination of these two).

So, different relative activations of muscles could be the best solution for generating an accurate force rather than for making an accurate movement. This observation can probably explain observations by Tax et al. (1990) and Miller et al. (1992). In these two studies, different relative activations of mono- and bi-articular muscles were reported comparing two tasks which required the same external force (in the experiment of Tax et al. (1990), also the same external work), but the subjects had to control different variables: either the force or the movement. 
To make predictions on the basis of the sensitivity of muscle sets, the sensitivity requirements of a task have to be defined. In the experiments of Tax et al. (1990) and Miller et al. (1992), no accuracy constraint was given: the direction of force and movement was not measured (and was partly constrained by the experimental set-up), and the speed of movement and the rate of force increase was chosen freely by the subjects.

Experiments which are more easily described in terms of accuracy constraints are those of Goossens et al. (1993). In their experiment, two isometric conditions with different accuracy constraints were compared. In both conditions, the rate of force increase was chosen by the subjects, whereas the direction of force was prescribed by the experimenter. In one condition, the subjects had no visual feedback of the actual force direction, whereas in the second condition, the subjects could see the direction (and amplitude) of their exerted force on an oscilloscope. Their result was that the relative contribution of bi-articular muscles was larger in the condition without visual feedback than in the condition with visual feedback.

For a theoretical prediction of the result of Goossens et al. (1993), the requirements for the sensitivity have to be determined. With a high directional sensitivity, small changes in muscle force lead to large changes in direction. This is a disastrous strategy without visual feedback, whereas with visual feedback the directional errors can be corrected. One would therefore expect that subjects would use the most robust strategy (low sensitivity) in the condition without visual feedback, and probably a more sensitive strategy in the condition with visual feedback. According to Fig. $5 \mathrm{~B}$, this would predict for most directions a bi-articular strategy for the condition without visual feedback, and a mono-articular strategy for the condition with visual feedback. This is indeed the experimental result of Goossens et al. (1993).

\section{Sensory-motor integration}

Apart from accurately sensing and acting, the human motor system can deal with the variability in its environment in an astonishingly flexible way. We never have any problems with the various masses of our shoes, or the differences in mass of our coffee-cup during the process of drinking its contents. How do we do this? What mechanisms exist to compensate for thcse misjudgements?

In textbooks on physiology, one can find two mechanisms which are 
responsible for this compensation. On the one hand, there is a passive mechanical compensation by the force-velocity relationship and stiffness properties of the muscles. On the other hand, there is an active compensation by the stretch reflex. In those textbooks, however, only single-joint situations are regarded. How do these mechanisms work in the multi-joint case, and what is the role of bi-articular muscles in these mechanisms?

In the center panel of Fig. 1, if the arm is displaced slightly to the right, only the flexors of the shoulder are stretched. The stretched shoulder flexor will generate a moment which leads to a force parallel to the lower arm. This reaction does not counteract the perturbation very well: the direction of the reaction is $45^{\circ}$ misaligned. If bi-articular muscles are present (right panel of Fig. 1), a displacement to the right stretches also biarticular flexors, which causes an elbow flexing moment, leading to a better directed reaction force. Hogan (1985) showed that in some situations, bi-articular muscles can even make the reaction force exactly counteract the perturbation.

The response to small perturbations of movement execution is very important in normal motor performance. Or to put it in another way: for a good performance, the sensori-motor system should be insensitive to slight misjudgements of load, starting position, etc. In simulation studies, Van Soest et al. (1992) and Van Soest and Bobbert (1993) showed the importance of both the passive properties and the bi-articularity of muscles for an explosive movement as vertical jumping. Although not explicitly tested, the passive properties of the bi-articular muscles seem to be an important factor in compensating for small perturbations.

Apart from this passive mechanism an active compensation is also used for correcting for misjudgements. Loss of proprioceptive information therefore severely impairs the co-ordination of multi-joint movements (Ghez et al., 1990). The multi-joint behaviour of the active compensation has been studied by Smeets et al. (1990) and Smeets and Erkelens (1991). A misjudgement of load for a fast elbow flexion movement for instance leads to considerable changes in the EMG activity of both agonists and antagonists (Smects ct al., 1990). To make an clbow flexion movement, cqual moments about elbow and shoulder are needed. A correction for a misjudgement of load therefore requires equal corrections of the moments about these joints. So, a co-ordinated compensation would yield equal EMG changes for muscles around both joints. The EMG corrections Smeets et al. (1990) reported were indeed equal for both joints.

How are the muscles co-ordinated to correct adequately? Smeets and 
Erkelens (1991) showed that at least part of this mechanism is at a spinal level: a short latency stretch reflex is also found in muscles that are not stretched themselves but whose activation helps to counteract the perturbation. This reflex must be caused by the stretch response of spindles in muscles crossing the moving joint. Whether the bi-articular muscles have an important role in this cannot be concluded from these experiments. Smeets and Denier van der Gon (1994) showed that connections between spindle-afferents and motoneurones can develop by a Hebbian learning mechanism from the correlation between activity of muscle spindles and motoneurones. According to their calculations, there will be no monosynaptic spinal connections from spindles of mono-articular flexors to motoneurons of mono-articular flexors of other joints. If these model predictions are true, then the responses of bi-articular muscle spindles are the only source of information available for co-ordinated compensation in the situations tested by Smeets et al. (1990) and Smeets and Erkelens (1991).

\section{General discussion}

A full model study of the accuracy of arm movements would include variations in joint-angle, moment arms, etc. Although these aspects are beyond the scope of this paper, I will discuss some of them shortly. A first comment is that when the elbow is almost completely flexed, the performance of the mono-articular set is clearly better than that of the bi-articular set. A more important aspect is the definition of accuracy as an overall performance. This is useful to investigate a general strategy. For a single movement, however, its own requirements will determine whether a high sensitivity is useful or not.

The sensory aspects are studied assuming a constant gain of the muscle spindles. During natural movements, however, the activation of intrafusal muscle fibres will change this gain, and thus the sensitivity. To compare controlling movements to controlling force, one has to make assumptions about the effect of muscle activation on spindle sensitivity. Further modelling effort is not very useful without experimental data in which the accuracy constraints are better defined as in the experiments by Tax et al. (1990) and Miller et al. (1992).

In this paper, an approach to study the effect of the geometry of the limb and muscle attachment on the accuracy of movements is described. Using an extremely simplified model, the use of bi-articular muscles 
instead of mono-articular elbow flexors and extensors showed to be advantageous. Accuracy considerations put an additional constraint on muscle coordination. This can explain differences in control strategies between situations which do not differ biomechanically from each other.

\section{References}

Ghez, C., J. Gordon, M.F. Ghilardi, C.N. Christakos and S.E. Cooper, 1990. Roles of proprioceptive input in the programming of arm trajectories. Cold Spring Harbor Symposia in Quantitative Biology $55,837-847$.

Goossens, H.H.L.M., J.B.J Smeets and C.J. Erkelens, 1993. Different relative activations of Mono- and bi-articular muscles in two isometric force tasks (submitted for publication).

Hogan, N., 1985. The mechanics of multi-joint Posture and movement control. Biological Cybernetics $52,315-331$.

Miller, L.E., C.C.A.M. Gielen, M. Theeuwen and C. Doorenbosch, 1992. 'The activation of mono- and biarticular muscles in multijoint movements'. In: R. Camminiti, P.B. Johnson and Y. Burnod (Eds.), Control of arm movement in space, (pp. 1-16). Berlin: Springer-Verlag.

Rothwell, J.C., M.M. Traub, B.L. Day, J.A. Obeso, P.K. Thomas and C.D. Marsden, 1982. Manual motor performance in a deafferented man. Brain 105, 515-542.

Sittig, A.C., J.J. Denier van der Gon and C.C.A.M. Gielen, 1985. Separate control of arm Position and velocity demonstrated by vibration of muscle tendon in man. Experimental Brain Research 60 , $445-453$.

Smeets, J.B.J. and J.J. Denier van der Gon, 1994. An unsupervised neural network for the development of reflex co-ordination. Biological Cybernetics 70, 417-425.

Smeets, J.B.J. and C.J. Erkelens, 1991. Dependence of Autogenic and heterogenic stretch reflexes on preload activity in the human arm. Journal of Physiology 440, 455-465.

Smeets, J.B.J., C.J. Erkelens and J.J. Denier van der Gon, 1990. Adjustments of fast goal-directed movements in response to an unexpected inertial load. Experimental Brain Research 81, 303-312.

Stein, R.B., 1982. What muscle variables does the nervous system control in limb movements? Behavioral and Brain Sciences 5, 535-577.

Tax, A.A.M., J.J. Denier van der Gon and C.J. Erkelnes, 1990. Differences in coordination of elbow flexor muscles in force tasks and in movement tasks. Experimental Brain Research 81, 567-572.

Van Soest, A.J., A.L. Schwab, M.F. Bobbert and G.J. van Ingen Schenau, 1992. The influence of the bi-articularity of the gastrocnemius muscle on vertical jumping achievement. Journal of Biomechanics $26,1-8$.

Van Soest, A.J. and M.F. Bobbert, 1993. The contribution of musclc properties in the control of explosive movements. Biological Cybernetics 69, 195-204. 